\title{
Casa da qualidade e qualidade da informação: revisão sistemática
}

Fernanda dos Santos

\author{
Doutoranda em Engenharia e Gestão do \\ Conhecimento pela Universidade Federal de Santa \\ Catarina
}

Lia Caetano Bastos

Doutora em Engenharia de Produção. Professora da Universidade Federal de Santa Catarina

http://dx.doi.org/10.1590/1981-5344/2731

As informações são consideradas matéria-prima para criação de conhecimento, este que por sua vez agrega valor às organizações, proporciona inovação e dá destaque para as organizações. Para que as organizações se mantenham competitivas na Era do Conhecimento, é necessário que tenham atenção à qualidade da informação estratégica, buscando formas de avaliá-las, proporcionando, por consequência, maior assertividade nas tomadas de decisão. Com isso, esta pesquisa buscou através de uma revisão sistemática de literatura compreender de que forma a Casa da Qualidade, matriz do método QFD (Quality Function Deployment) tem interagido com a qualidade da informação. Embora com poucas pesquisas ainda relatando esta interação, o método QFD, um dos métodos trabalhados na gestão da qualidade total, mostra-se como uma ferramenta que pode auxiliar as organizações na avaliação da qualidade da informação organizacional através de adaptações de sua versão original.

Palavras-chave: Qualidade da informação; Casa da Qualidade; Informação estratégica organizacional.

\section{House of quality and information quality: systematic review}

Information is considered material for knowledge creation, this in turn adds value to organizations providing innovation and highlights for organizations. For 
organizations to remain competitive in the Knowledge society, you need to have attention to the quality of strategic information, looking for ways to evaluate them in order to ensure that they provide greater assertiveness in decision making. Thus, this research sought through a systematic literature review to understand how the house of quality, matrix of QFD (Quality Function Deployment) method has interacted with the information quality. Although with little research still reporting this interaction, the QFD method, one of the methods worked on total quality management, it is shown as a tool that can assist organizations in assessing the organizational information quality through adaptation of the original version.

Keywords: Information quality; House of Quality; Organizational strategic information.

Recebido em 03.03.2016 Aceito em 03.02.2017

\section{Introdução}

Viver na Era do Conhecimento significa viver em busca de inovação, informações com qualidade e conhecimentos com alto valor agregado que possam levar as empresas e pessoas a ter destaque frente ao mundo altamente competitivo e globalizado.

Segundo destacam Fialho et al. (2010) nesta era, a informação e o conhecimento se tornam "armas competitivas", e por isso, as organizações necessitam estar atentas para a construção de uma base de seus conhecimentos.

O excesso de informações pode levar a empresa à desinformação (DALFOVO, 2007). Com isso, é necessário o cuidado com a qualidade da informação que esta transitando. Quando definidas as informações estratégicas, faz-se necessário certificar que tenham qualidade, e que permitam que as decisões não tragam prejuízos ao invés de benefícios.

De acordo com Fialho et al. (2010, p. 43) "a informação é um meio ou material necessário para extrair e construir o conhecimento, alterandoo por meio do acréscimo de algo ou reestruturando-o". Tão importante quanto garantir a tecnologia adequada para armazenar, compartilhar e recuperar o conhecimento é garantir que ele não sofrerá redução semântica. O conhecimento precisa ser concebido, armazenado e compartilhado de maneira que esteja alheio aos diversos contextos em que circular e independente do emissor e do receptor que o utilizarão (GOMEZ, 1993).

Santos e Valentim (2015) consideram que a informação organizacional, aquela que está voltada para o desenvolvimento do negócio, precisa ser corretamente gerida, pois é a base no desenvolvimento de novos produtos, melhoria na qualidade da 
produtividade, melhoria nos serviços ofertados, e garantia de tomadas de decisão mais acertadas.

Dalfovo (2007, p. 60) trás que "A gestão do conhecimento não se aplica, se pratica; está pautada na coerência e atitude dos gerentes e funcionários, na aprendizagem e compartilhamento das ideias". E seguindo nesta linha de entendimento de fluxo de informações dentro da organização, Moura et al. (2014, p. 104) destacam que "A boa execução das estratégias eleitas, ao transformá-las em ações de fato estratégicas, dependerá de como é aceita e apreendida pelas pessoas que formam a organização".

Enquanto Woiciekovski e Pereira (2015) relatam que as organizações afoitas por utilizar o máximo dos dados e informações que possuem investem em tecnologia da informação, porém esquecem-se da capacitação das pessoas, orientando-as para que possam identificar quais são as informações estratégicas da organização, aquelas que realmente contribuirão para o aumento da competitividade organizacional, Teixeira e Valentim (2016, p. 7) destacam que "as informações precisam ser tratadas no contexto da organização, isto é, analisadas e compartilhadas pelo grupo de gestores, para se tornarem relevantes e de maneira concreta transformarem-se em ação".

Zarraga-rodriguez e Avarez (2015) salientam que muitos estudos já ocorrem relacionando a qualidade da informação com a satisfação do usuário, porém ainda poucos estudos relatam a qualidade da informação e seu uso tanto em nível individual quanto organizacional.

Ferramentas advindas da Gestão da Qualidade Total como PDCA, PSP, Espinha de Peixe, e Quality Function Deplyment (QFD) são utilizadas para análise da qualidade da informação. Neste sentido, a proposta deste estudo é através de uma pesquisa exploratória, utilizando revisão sistemática da literatura, compreender de que forma a ferramenta da Gestão da Qualidade Total QFD tem sido utilizada para análise da qualidade da informação.

\section{Métodos de pesquisa}

Esta pesquisa apresenta fins exploratórios, que de acordo com Freire (2013) visa, através de levantamentos bibliográficos, proporcionar uma aproximação com o tema e recuperar informações disponíveis.

A Revisão Sistemática da Literatura (RSL) é uma técnica de levantamento de dados que segue passos sistemáticos buscando identificar pontos específicos sobre determinado tema de pesquisa ou área pretendida (FREIRE, 2013). Com isso, esta pesquisa ocorre utilizando o método de RSL, seguindo os passos apresentados na Figura 1. 


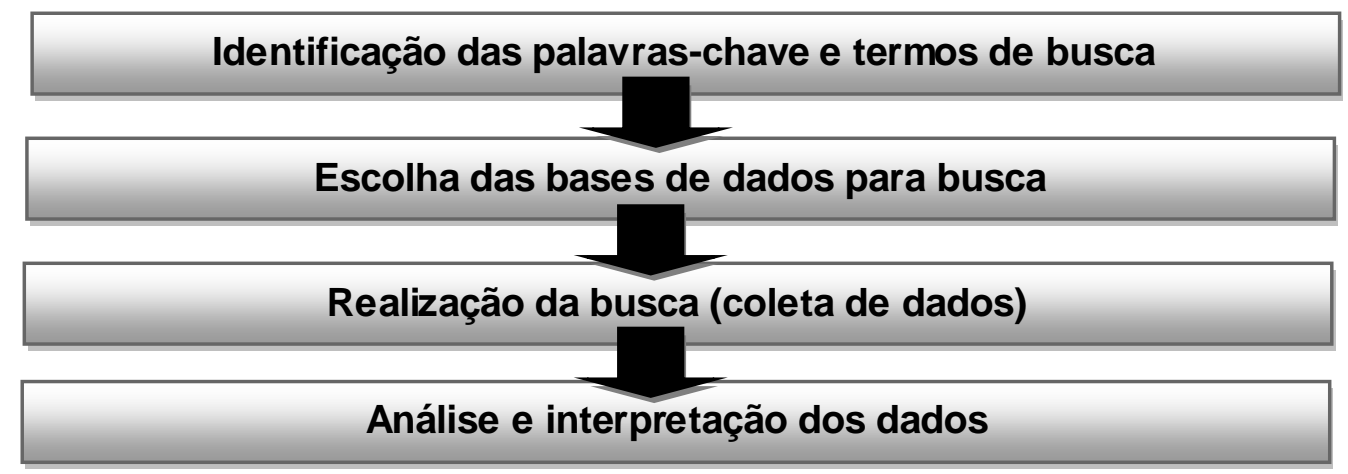

Figura 1 - Passos realizados para RSL

Fonte: Dados da pesquisa.

A análise dos dados ocorreu com base na Bibliometria, a qual, conforme Araujo et al. (2000), busca observar a evolução da literatura e o conhecimento produzido no decorrer dos anos. Conforme relata Freire (2013), a bibliometria é baseada em Leis que devem ser observadas e seguidas, quando se utiliza o método, quais sejam: Lei de Lotka (1926) medição da produtividade de autores; Lei de Bradford (1934) - medição da produtividade do assunto em um conjunto de revistas; e Lei de Zipf (1949) - medição da frequência de aparecimento de palavras e termos.

Dentre as métricas que podem ser analisadas quando se utiliza a bibliometria e citadas por Freire (2013), esta pesquisa apresenta: total de publicações, relevância de citações, número de publicações por autor e número de publicações por periódico.

Para tal, a pesquisa foi realizada na base de dados internacional SCOPUS, buscando pelos termos "Information Quality", "QFD" e "House Of Quality", em título, resumo e palavras-chave, utilizando o auxilio dos conectores booleanos AND e OR, sendo, portanto: TITLE-ABSKEY ("Information quality" AND "QFD" OR "House of Quality").

\section{Apresentação e análise dos resultados}

A busca retornou 15 documentos, sendo destes: Conference Paper (8); Article (6) e Book Chapter (1). Os documentos foram publicados entre os anos de 2006 e 2015, sendo do ano de 2006 o documento mais citado - dos autores Xu e Yan com o título "An intelligent estimation method for product design time", citado dezoito vezes. 


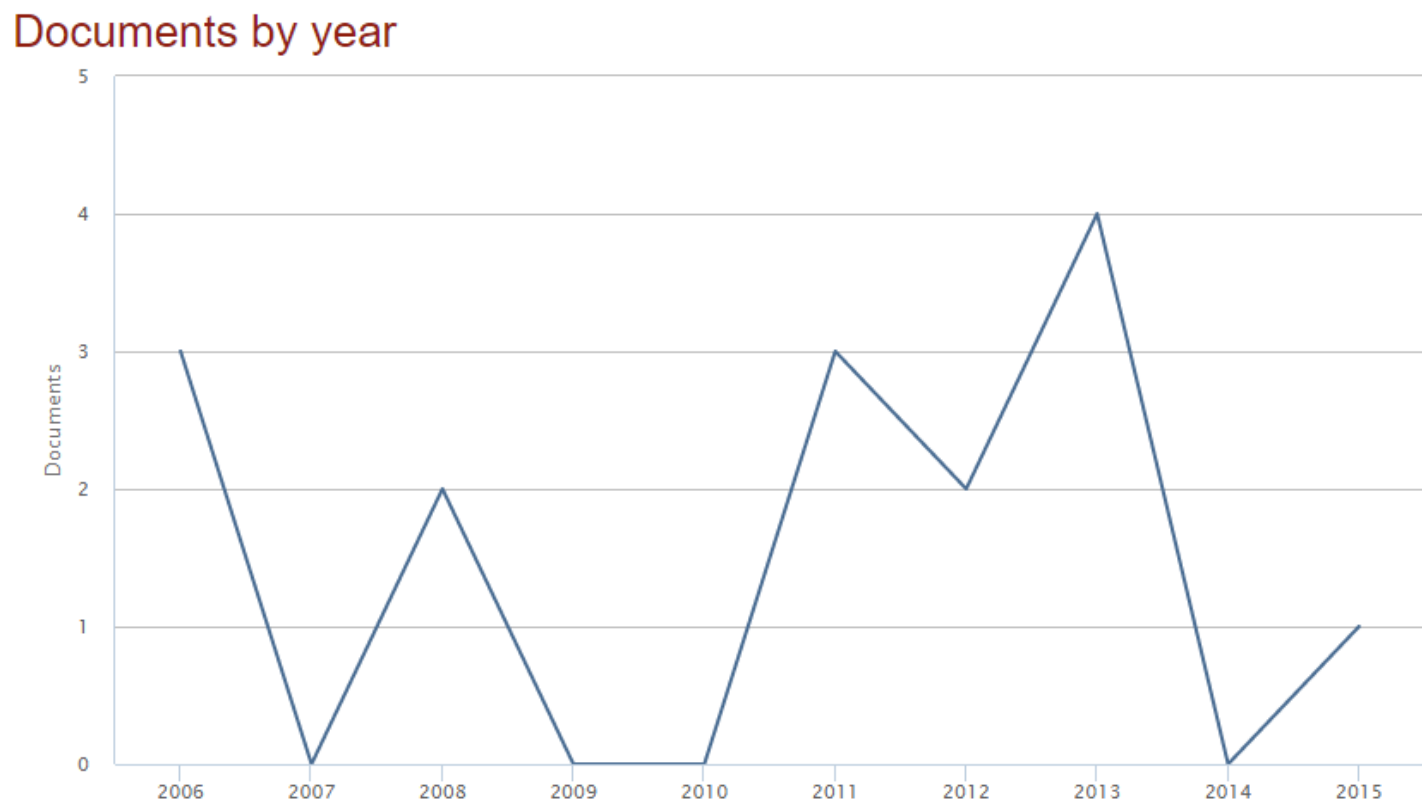

Gráfico 1- Publicações por ano

Fonte: SCOPUS (2016).

Apresentando picos de publicação entre os anos, 2014 é o ano que apresenta maior número de publicações sobre o tema, com a quantidade de quatro documentos. Os anos de 2007, 2009, 2010 e 2014 não apresentam registros de publicações.

$\mathrm{Na}$ análise de publicações por autor, quarenta e um autores participam como autor ou co-autor dos documentos, sendo Zang o único que apresenta participação em dois documentos, conforme mostra o Gráfico 2.

Documents by author

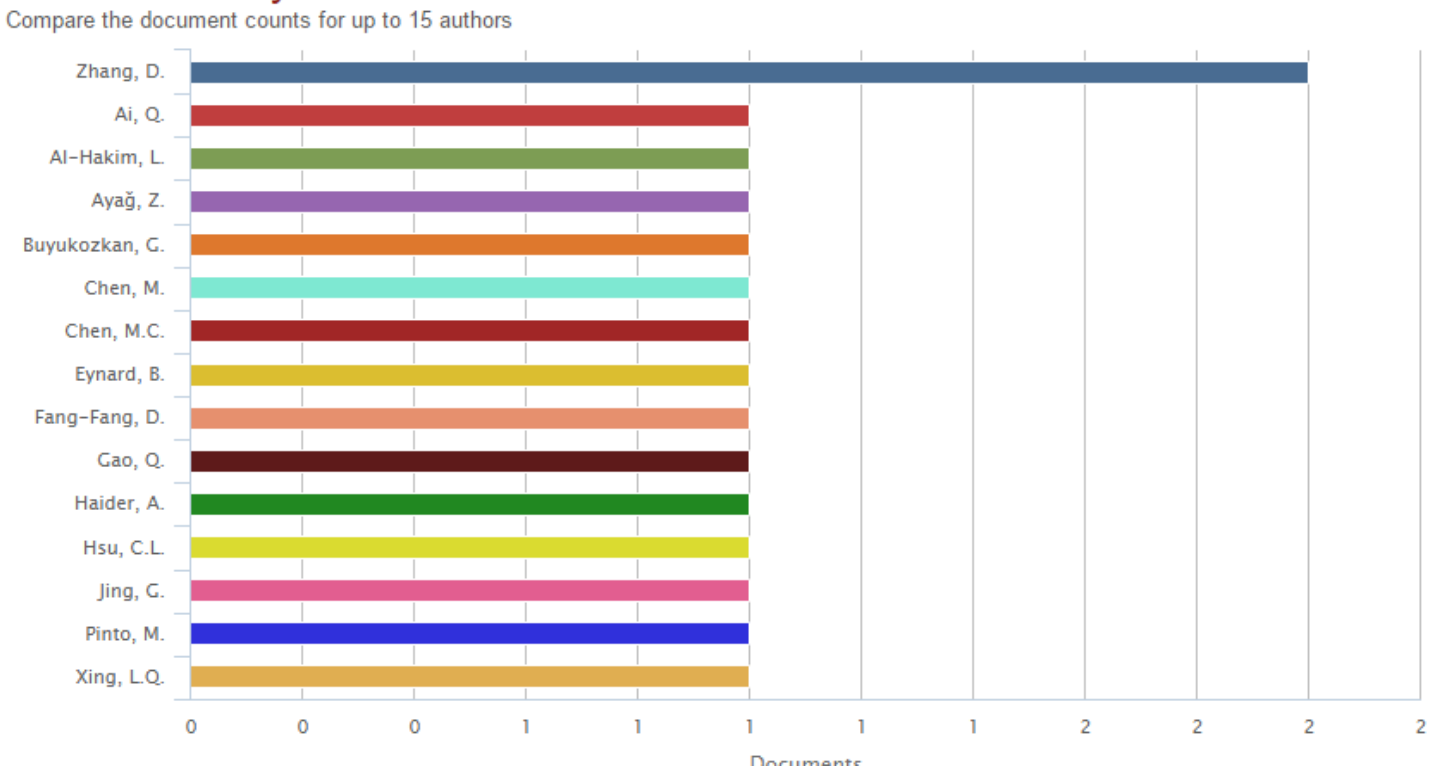

Gráfico 2 - Publicações por autor

Fonte: SCOPUS (2016). 
O capitulo de livro encontrado na busca - "Information Quality Function Deployment" - faz parte da obra "Challenges of Managing Information Quality in Service Organizations" do autor Latif Al-Hakim do ano de 2006. Tanto articles quanto conference papers apresentam publicações em diferentes fontes, sendo demonstrado no Quadro 1.

\section{Quadro 1 - Fontes de publicação}

\begin{tabular}{|c|c|}
\hline \multirow{8}{*}{ Articles } & Advanced Materials Research \\
\hline & Enterprise Information Systems \\
\hline & Expert Systems with Applications \\
\hline & International Joumal of Advanced Manufacturing Technology \\
\hline & Journal of Information Science \\
\hline & Journal of Intelligent Manufacturing \\
\hline & Journal of Systems Engineering and Electronics \\
\hline & Lecture Notes in Business Information Processing \\
\hline \multirow{6}{*}{$\begin{array}{l}\text { Conference } \\
\text { Paper }\end{array}$} & $\begin{array}{l}\text { International Conference on Consumer Electronics, Communications and Networks, CECNet } \\
2011 \text { - Proceedings }\end{array}$ \\
\hline & Chinese Control and Decision Conference, 2008, CCDC 2008 \\
\hline & 19th International Conference on Industrial Engineering and Engineering Management 2013 \\
\hline & IEEE International Conference on Industrial Engineering and Engineering Management 2012 \\
\hline & $\begin{array}{l}\text { TCEIS } 2011 \text { - Proceedings of the 13th International Conference on Enterprise Information } \\
\text { Systems }\end{array}$ \\
\hline & $\begin{array}{l}\text { IEEE International Conference on Industrial Engineering and Engineering Management, } \\
\text { IEEM } 2008\end{array}$ \\
\hline
\end{tabular}

Fonte: Dados da pesquisa.

Após análise dos resumos, quatro artigos foram selecionados para leitura integral e análise do conteúdo. Ao realizar uma busca complementar com o auxilio da ferramenta Google Acadêmico, mais um artigo foi identificado e selecionado. O Quadro 2 relaciona título, autores, ano e palavras-chave dos referidos documentos.

Quadro 2 - Lista dos artigos que relacionam QFD e Qualidade da Informação

\begin{tabular}{c|c|c|c}
\hline Título & Autor (es) & Ano & Palavras-chave \\
\hline \hline $\begin{array}{c}\text { Information Quality Function } \\
\text { Deployment }\end{array}$ & Latif Al-Hakim & 2004 & Information quality, information orientation, \\
QFD
\end{tabular}

Fonte: Dados da pesquisa.

O artigo "Information Quality Function Deployment" de Al-Hakim, adapta o QFD para encontrar as correlações entre dimensões dos sistemas de qualidade da informação e indicadores de desempenho e medidas dos sistemas de informação. Apresenta um estudo de caso realizado em um hospital, considerando que as informações relacionadas à área da saúde 
ainda não possuem a atenção devida em relação ao seu grau de importância nas tomadas de decisão que Ihe são atribuídas.

Em uma entrevista inicial, Al-Hakim (2004) ouviu a voz do consumidor, primeiro passo para a construção da Casa da Qualidade no método QFD. Entrevistou para tal, profissionais do hospital e pediu que indicassem dentre as dimensões da qualidade da informação aquelas que sugerem ter maior importância na utilização de um sistema de Tecnologia da Informação (TI).

Em uma segunda etapa, relacionou as dimensões destacadas pelos usuários do sistema e pediu aos profissionais da área de TI que as relacionassem com as medidas de orientação da informação, baseadas Marchand et al. (2000), que são Práticas para TI, Práticas para gestão da informação, e Valores e comportamentos da informação, conforme ilustra a Figura 2.

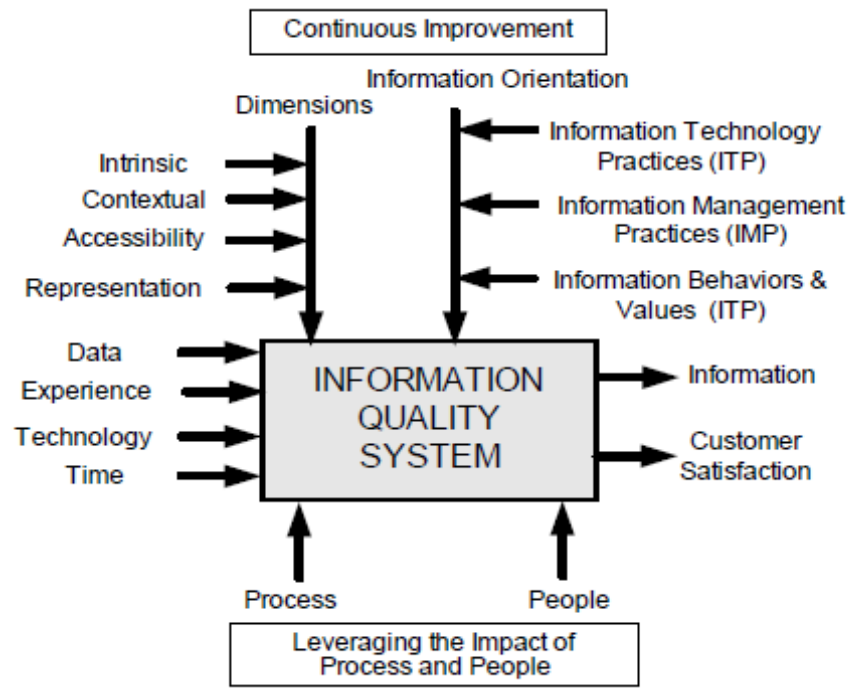

Figura 2 - Processo de melhoria contínua da Informação

Fonte: AL-HAKIM (2004, p. 175).

Em uma nova rodada de correlações, foram analisados os itens de medidas de orientação a informação com outros itens de medidas de orientação a informação. Ao final, o resultado apresentou cinco medidas nas quais a gestão do hospital deve ter mais atenção para que consiga melhorar a qualidade da informação.

Pinto (2006) apresenta a informação como um produto e propõe em seu artigo, utilizar o QFD para identificar os principais fatores e dimensões que precisam ser considerados na hora da produção de produtos da informação e processos de representação para garantia da qualidade.

A primeira etapa da pesquisa foi conseguir identificar os requisitos considerados de maior relevância pelos usuários, onde se destacaram três características: acesso às informações criticas; coleta das informações criticas; e atualização das informações criticas. O passo seguinte apresentado pelo autor é a elaboração da Casa da Qualidade. As características de qualidade destacadas pelos especialistas e com as quais 
se busca a correlação no primeiro momento são: representação, relação, filtro e a busca por fontes relevantes.

$\mathrm{Na}$ sequencia são analisadas as dimensões que tem relação com a integração das informações, sendo consideradas as características de qualidade: relevância, consistência, acurácia, abrangência, formato e atualidade. Os passos seguintes da pesquisa apresentados no artigo são as relações que direcionam para as características da qualidade nos processos de representação.

Wang et al. (2008) destacam que o QFD e a Casa da Qualidade são ferramentas eficazes no auxilio da tradução da voz do consumidor em características específicas para o melhoramento da qualidade da informação. Os autores utilizam a Casa da Qualidade para verificar as dimensões da Qualidade da Informação que precisam ser vistas com mais atenção em uma empresa industrial.

Neste estudo, os autores relatam a informação como um produto, podendo, portanto, ser coletado, armazenado, organizado e transformado. Destacam que utilizar a Casa da Qualidade para a Qualidade da Informação segue basicamente os mesmos princípios da aplicação para materiais na indústria, porém algumas adaptações são necessárias, pois os requisitos da qualidade da informação apontados pelos consumidores são diferentes daqueles apontados para produtos industriais.

A Casa da qualidade foi construída com o cruzamento entre os requisitos da qualidade identificados e priorizados pelos consumidores a partir de três categorias: produção da informação, processamento e conteúdo da informação e, as dimensões da qualidade da informação destacadas por profissionais da informação.

Wang et al. (2008, p. 217) relatam que "com base nesta metodologia, os pontos fracos da qualidade da informação em empresas de manufatura podem ser identificados na forma de dimensões da qualidade da informação e ações para a melhoria da qualidade das informações podem ser realizadas" (tradução livre do autor). Ao final afirmam que seu estudo foi uma apresentação breve mostrando a possibilidade da aplicação do QFD no campo da qualidade da informação, mas salientam que mais estudos, com exemplos de aplicações na área são necessários.

É de Lin, Jing e Fang-Fang (2011) o artigo intitulado "Evaluation Method of Enterprise Information Quality Based on QFD" no qual a Casa da Qualidade é adaptada para a avaliação da qualidade da informação, como mostra a Figura 3. 


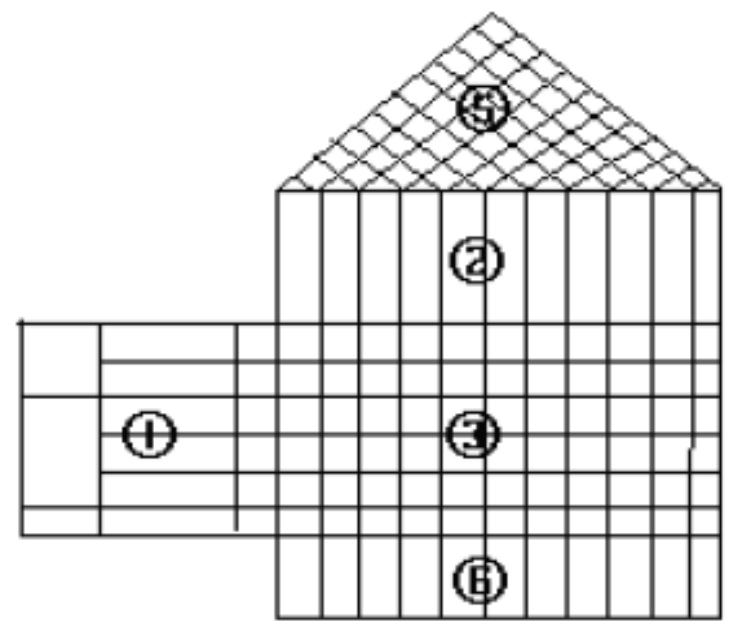

Figura 3 - Casa da Qualidade adaptada à Qualidade da Informação

Fonte: LIN; JING; FANG-FANG (2011, p. 327).

Nesta adaptação, a parede esquerda (1) apresenta o "indicador de qualidade de informação corporativa e seu grau de importância", o limite máximo (2) apresenta as dimensões da qualidade da informação, o quarto (3) mostra a matriz de relação entre indicador de qualidade de informação corporativa e as dimensões da qualidade da informação. O telhado (5) irá mostrar a inter-relação entre os indicadores da qualidade da informação e o piso (6) apresenta o peso das dimensões referentes à qualidade da informação da organização, onde podem ser identificados os gaps.

Conforme destacam Lin, Jing e Fang-Fang (2011), neste modelo de Casa da Qualidade adaptada à avaliação da informação organizacional é possível visualizar os indicadores de qualidade da informação e seu grau de importância (obtido com os usuários), as dimensões da qualidade da informação conforme seleção feita por equipe de especialistas, e os gargalos na qualidade da informação que esta sendo avaliada.

Lee e Haider (2012) também compreendem a informação como um produto e utilizam em seu artigo processo de hierarquia analítica na identificação da correlação entre as dimensões da qualidade da informação e o método QFD na determinação dos fatores fundamentais para a gestão da qualidade da informação.

A pesquisa foi aplicada em uma fábrica Coreana, para identificar a importância relativa das dimensões da qualidade da informação, os autores aplicaram questionário junto aos empregados da organização. Dentre os problemas de gestão e qualidade da informação extraídos dos questionários estão: dados duplicados, falta de padronização, diferentes formas de operar os sistemas de informação, informações com baixa consistência, e informações incompletas.

Lee e Haider (2012) destacam que "usuários, guardiões e gestores da informação percebem facilmente o impacto da qualidade, ou falta da qualidade da informação em seu trabalho". Na casa da qualidade construída por eles, a correlação ocorre entre os itens avaliados no 
questionário pelos usuários da informação e as dimensões da qualidade da informação divididas em duas categorias: Conformidade com a Especificação e Atende ou Excede as Expectativas dos consumidores. Estas por sua vez se subdividem em 2 categorias cada uma, tendo ao total 4 subcategorias e 15 dimensões da qualidade da informação, conforme demonstra o Quadro 3.

Quadro 3 - Dimensões da Qualidade utilizadas por Lee e Haider (2012)

\begin{tabular}{|c|c|c|}
\hline \multirow{6}{*}{ Conformidade com a Especificação } & \multirow{4}{*}{$\begin{array}{l}\text { Qualidade da informação } \\
\text { (produto) }\end{array}$} & Livre de erro \\
\hline & & Concisão \\
\hline & & Completude \\
\hline & & Consistência \\
\hline & \multirow{2}{*}{$\begin{array}{l}\text { Qualidade da informação } \\
\text { (serviço) }\end{array}$} & Atualidade \\
\hline & & Segurança \\
\hline \multirow{9}{*}{$\begin{array}{l}\text { Atende ou Excede as Expectativas dos } \\
\text { consumidores }\end{array}$} & \multirow{5}{*}{$\begin{array}{l}\text { Qualidade da informação } \\
\text { (produto) }\end{array}$} & Qualidade adequada \\
\hline & & Relevância \\
\hline & & Fácil entendimento \\
\hline & & Interpretabilidade \\
\hline & & Objetividade \\
\hline & \multirow{4}{*}{$\begin{array}{c}\text { Qualidade da informação } \\
\text { (serviço) }\end{array}$} & Credibilidade \\
\hline & & Acessibilidade \\
\hline & & $\begin{array}{l}\text { Facilidade de } \\
\text { operacão }\end{array}$ \\
\hline & & Reputação \\
\hline
\end{tabular}

Fonte: Dados da pesquisa.

Após a Casa da Qualidade e as correlações entre os requisitos dos consumidores e as dimensões da qualidade da informação, um diagrama da Causa e Efeito (Espinha de peixe) foi elaborado pelos gestores da informação da empresa em questão.

\section{Considerações finais}

A ferramenta Quality Function Development (QFD) pode ser compreendida como uma ferramenta analítica que irá quantificar através de matriz a relação entre as necessidades dos clientes e o processo de desenvolvimento de novos produtos. Uma de suas etapas consiste na elaboração da Casa da Qualidade, chamada assim por seu desenho que lembra uma casa. Ela permite compreender as principais contradições entre necessidades dos clientes e características técnicas, auxiliando no desenvolvimento de um projeto com qualidade desde a sua concepção. (LIN, JING, FANG-FANG, 2011).

Os artigos citados e analisados demonstram que a qualidade da informação enquanto área de pesquisa tem avançado nas últimas décadas, estimulado pelo entendimento de que a sociedade está na era do conhecimento, onde informações corretas e no tempo certo são fatores que agregam valor e elevam a importância deste tipo de pesquisa.

É perceptível que a informação pode ser trabalhada enquanto produto e enquanto processo, com a atenção devida para cada um dos personagens que trabalham com a informação: produtores, guardiões e consumidores da informação. Ouvir as necessidades do usuário e considerá-las na elaboração de planos de gestão da informação poderá 
poupar para as organizações custos com a má qualidade, seja no desenvolvimento e produção de produtos, ou na tomada de decisões.

Os artigos buscam analisar a qualidade da informação de acordo com as características de qualidade atribuídas pelos clientes, e as utilizam para análise da informação enquanto parte do processo de criação. Poucos autores trazem e analisam a informação enquanto produto, não ficando clara a utilização das características para mensurar a qualidade de informações pré-selecionadas.

Lin, Jing e Fang-Fang (2011) se aproximam deste modelo quando apresentam uma possibilidade de adaptação da Casa da qualidade para a análise da informação organizacional. Desta forma, evidencia-se que ainda existe carência na literatura sobre a demonstração de caso real em que se analise a qualidade da informação enquanto produto organizacional utilizando tal ferramenta. Assim, este estudo traz como propostas para estudos futuros a aplicação da Casa da Qualidade para a análise da qualidade da informação organizacional.

\section{Referência}

AL-HAKIM, L. Information quality function deployment. In: INTERNATIONAL CONFERENCE ON INFORMATION QUALITY, 9., 2004, Berkeley. Proceedings... Berkeley: Iciq, 2004. p. 170-182.

ARAUJO, W. T. et al. Meta-análise das dissertações do curso de mestrado ciência da informação UFPB: 1990- 1999. Revista Informação e Sociedade: estudos, João Pessoa, v. 10, n. 1, p. 1-11, 2000.

DALFOVO, O. Modelo de integração de um Sistema de Inteligência Competitiva com um Sistema de Gestão da Informação e de Conhecimento. 2007. 240f. Tese (Doutorado) - Curso de Engenharia e Gestão do Conhecimento, Programa de Pós-Graduação em Engenharia e Gestão do Conhecimento do Centro Tecnológico, Universidade Federal de Santa Catarina, Florianópolis, 2007.

FIALHO, F. et al. Gestão do conhecimento organizacional. Florianópolis: Editora UfSc, 2010. 200 p.

FREIRE, P. de S. Aumente a qualidade e quantidade de suas publicações científicas: manual para elaboração de projetos e artigos científicos. Curitiba: CRV, 2013. 90 p.

GOMEZ, M. N. G. de. A representação do conhecimento e o conhecimento da representação: algumas questões epistemológicas. Ci. Inf., Brasília, v. 22, n. 3, p. 217-222, set./dez. 1993.

LEE, S. H.; HAIDER, A. Assessing information quality by Six Sigma Method. Dasfaa Workshops, Springer-verlag Berlin Heidelberg, p. 323334, 2012.

LIN, G.; JING, G.; FANG-FANG, D. Evaluation method of enterprise information quality based on QFD. Ieee: International Conference on 
Consumer Electronics, Communications and Networks (CECNet), Xianning, v. 1, n. 1, p. 325-328, Apr. 2011.

MARCHAND, D. A.; KETTINGER, W. J.; ROLLINS, J. D. Information orientation: people, technology and the bottom line. Magazine: Summer, [s. L.], p. 1-17, 15 jul. 2000.

MOURA, G. L. de et al. Integração entre pesquisa e desenvolvimento e planejamento estratégico: um estudo em montadoras automobilísticas. Gestão \& Regionalidade, São Caetano do Sul, v. 30, n. 89, p. 102-115, maio/ago. 2014.

PINTO, M. Data representation factors and dimensions from the quality function deployment (QFD) perspective. Journal of Information Science, [s.I.], v. 32, n. 2, p.116-130, 1 abr. 2006.

SANTOS, J. C. dos; VALENTIM, M. L. P. Gestão da informação em ambientes organizacionais: em foco o setor têxtil e de vestuário. Inf. Prof., Londrina, v. 4, n. 1, p. 56-81, jan./jun. 2015.

SCOPUS. 2016. Disponível em: <https://www.scopus.com/>. Acesso em: 9 fev. 2016.

TEIXEIRA, T. M. C.; VALENTIM, M. Lígia P. Inteligência Competitiva Organizacional: Um Estudo Teórico. Perspectivas em Gestão \& Conhecimento, João Pessoa, v. 6, n. Especial, p. 3-15, jan. 2016.

WANG, K. et al. Analysis of consumers' requirements for data/information quality by using HOQ. In: IEEE INTERNATIONAL CONFERENCE ON INDUSTRIAL ENGINEERING AND ENGINEERING MANAGEMENT, 2008, Singapore. Proceedings of the 2008 IEEE IEEM. Singapore: IEE, 2008. p. $213-217$.

WOICIEKOVSKI, E.; PEREIRA, Li. Inovação e estratégia sustentadas pelo gerenciamento de informações: um estudo nas empresas incubadoras na ITFETEP. Int. J. Knowl. Eng. Manage: IJKEM, Forianópolis, v. 4, n. 10, p. 125-142, nov./fev. 2016.

ZARRAGA-RODRIGUEZ, M.; ALVAREZ, M. J. Experience. Journal Of Data And Information Quality, [s.l.], v. 6, n. 2-3, p. 1-14, 8 jul. 2015. Association for Computing Machinery (ACM). DOI: 10.1145/2774223. 